\title{
Magnetic support of stellar slingshot prominences
}

\author{
Rose F. P. Waugh ${ }^{\circledR \star}$ and Moira M. Jardine \\ SUPA, School of Physics and Astronomy, University of St Andrews, North Haugh, St Andrews KY16 9SS, UK
}

Accepted 2018 November 16. Received 2018 November 15; in original form 2018 September 20

\begin{abstract}
We present models for the magnetic support of the 'slingshot prominences' observed in the coronae of rapidly rotating stars. We calculate mechanical equilibria of loops in a spherical geometry. Prominence-forming loops are found first for dipolar and quadrupolar stellar fields that are fully closed. Equilibria are then found within the stellar wind for a dipolar field that becomes open beyond a given radius. We identify two physical processes that may produce gaps in the distribution of prominence heights: the location of this opening radius, and the behaviour of the buoyancy force. The buoyancy may differ from one prominence-bearing loop to another if they are at different temperatures, thus potentially smearing out any gap in observed height distributions. We produce synthetic prominence distributions and compare to the observations of two well-observed stars: AB Doradus and Speedy Mic. The model recovers the more compact prominence distribution observed for Speedy Mic and reproduces better the overall shape of the height distributions for both stars when the opening radius is beyond the co-rotation radius.
\end{abstract}

Key words: stars: individual: AB Dor-stars: low mass-stars: magnetic field.

\section{INTRODUCTION}

Prominences are cool condensations of neutral hydrogen, embedded within a hot corona. These features are perhaps best known as dark filamentary structures against the solar disc, or bright material protruding above the solar limb. They are not restricted to our Sun, however, having been observed on many other stars albeit not in the same resolution. Since they are confined by the magnetic field, they trace out the coronal magnetic field structure. This makes them useful since the current method of generating such information involves extrapolation of the stellar surface magnetic field, gained from Zeeman Doppler imaging. These features could have consequences for stellar evolution. Ejection of active prominences is thought to be a method of stellar angular momentum and mass-loss (Hussain 2013). While the effect of these features will likely be small while compared to the stellar wind, it could be non-negligible (Aarnio, Matt \& Strassun 2012; Cranmer 2017; Odert et al. 2017). Ejections could also be responsible for loss of helicity from the system, as on the Sun (Low 2006, 1994; Wang, Zhou \& Zhang 2004). Development of any nearby planets, and their ability to harbour life, could also be influenced by ejection of these condensations. Impact of ejected prominences with an exoplanet could lead to geomagnetic storms, compression of the planetary magnetosphere by shock waves (Gonzalez et al. 1994), and atmospheric erosion due to the stripping of the planetary atmosphere by frequent impacts (Khodachenko et al. 2007).

^E-mail: rw47@st-andrews.ac.uk
The first suggestion of prominences on other stars came from binary systems where, as one star eclipsed its partner, the light from the background star was used to probe the atmosphere of the foreground star (Schroder 1983). When analysing the spectra, unexplained absorption features were found immediately preceding or subsequent to the rotation phase at which the eclipse took place. These transient absorption features had been seen to reoccur on consecutive periods, sometimes dimming or brightening in intensity. This suggested the material being supported in co-rotation could, in some cases, be reasonably stable. The star SS Bootis was observed by Hall et al. (1990) in 1987 and 1988, and then again in 1992 by Hall \& Ramsey (1992) as part of a larger scale study into these features. The authors reported the existence of a feature at the same height in both works. They suggested that either the feature was incredibly stable or that this could be a particularly stable point within the magnetic field, with condensations being repeatedly trapped at this point.

Similar absorption features were subsequently seen on the rapidly rotating, K0V type star, AB Doradus (Cameron \& Robinson 1989). They were observed as transient dips in the $\mathrm{H}_{\alpha}$ profiles, crossing the disc in a matter of hours and sometimes reappearing on consecutive nights. This behaviour was consistent with absorbing material located around two to nine stellar radii from the stellar rotation axis and co-rotating with the star (Cameron \& Robinson 1989). Thought to be held in co-rotation by the coronal magnetic field, these features were coined 'slingshot prominences' (Cameron 1996; Steeghs et al. 1996). While AB Dor may be the best observed rapid-rotator prominence host, prominences have been observed on multiple rapidly rotating stars(Dunstone et al. 2006a,b; Jeffries 1993; Byrne, Eibe \& Rolleston 1996; Barnes et al. 1998; Cameron \& 
Woods 1992; Barnes et al. 2000; Leitzinger et al. 2016; Skelly et al. 2008, 2009, 2010; Eibe 1998).

Slingshot prominences have also been seen on M dwarfs, for example V374 Peg (Vida et al. 2016), a fully convective, ultrafast rotator. Later, Stauffer et al. (2017) found 19 M dwarfs exhibiting prominence-like absorption dips within K2 light curves. Variations in flux that repeated at the same phase on each rotation were consistent with cloud condensations trapped in co-rotation. These stellar prominences on rapidly rotating stars are rather different to their solar namesakes. They typically occult a larger area of the stellar surface than solar prominences, are 10-100 times larger in mass and can be found significantly further out from the stellar rotation axis (Cameron 1999).

Previously, Jardine \& Cameron (1991) published a model to describe prominence formation within the equatorial plane of such rapidly rotating stars. The authors showed that for an isothermal atmosphere in hydrostatic equilibrium, a cool loop may reach a mechanical equilibrium whereby a prominence may be supported. This is achieved through the balance of the gravitational, Lorentz and centrifugal forces. Ferreira (2000) later published a paper discussing the stability of such prominences and Jardine \& van Ballegooijen (2005) found available equilibria within the stellar wind. This removed the requirement for the stellar corona to be closed up to the large heights from the stellar surface at which prominences are typically observed. More recently, Villarreal D'Angelo, Jardine \& See (2018) categorized which low-mass stars could be capable of supporting such prominences. By comparing whether the Alfvén radii of stars were inside or outside of the co-rotation radius, the authors were able to determine if prominence formation could be supported. This work adopted terms such as 'centrifugally supported magnetospheres' and 'dynamical magnetospheres', previously applied in the context of massive stars (Petit et al. 2013; Owocki et al. 2016). These terms are used to distinguish between distinct types of phenomena by which material is either supported against gravity or will fall back to the stellar surface. This idea of supporting material beyond the Keplerian co-rotation radius under force balance has been extensively discussed (Cassinelli et al. 2002; Townsend \& Owocki 2005; ud-Doula, Owocki \& Townsend 2008).

In this paper we aim to extend the model developed by Jardine \& van Ballegooijen (2005) of prominence formation in a locally twodimensional, Cartesian geometry. Here we extend their model to a spherical coordinate system, describing the low-order multipoles that dominate the magnetic field structure at the heights of several stellar radii at which these prominences are supported. Most implementations of the Zeeman-Doppler imaging technique express the recovered fields in terms of spherical harmonics and so can provide insight into the contribution of the different modes (Donati \& Landstreet 2009). In particular, we aim to show that solutions for mechanical equilibria in this geometry replicates the results of Jardine \& van Ballegooijen (2005) for prominence formation within the open field region. We consider the influence of the background field topology, as well as the impact of allowing the field to become open above and below the co-rotation radius. Since there is no direct observational evidence of the location at which the stellar magnetic field becomes open, we consider both cases here.

\section{METHOD}

The physical scenario that we model is that of cooled loops embedded in a hot external magnetic field. We choose both dipolar and quadrupolar external fields, and in both cases the magnetic axes lie in the equatorial plane of the star. In the case of the dipolar field,
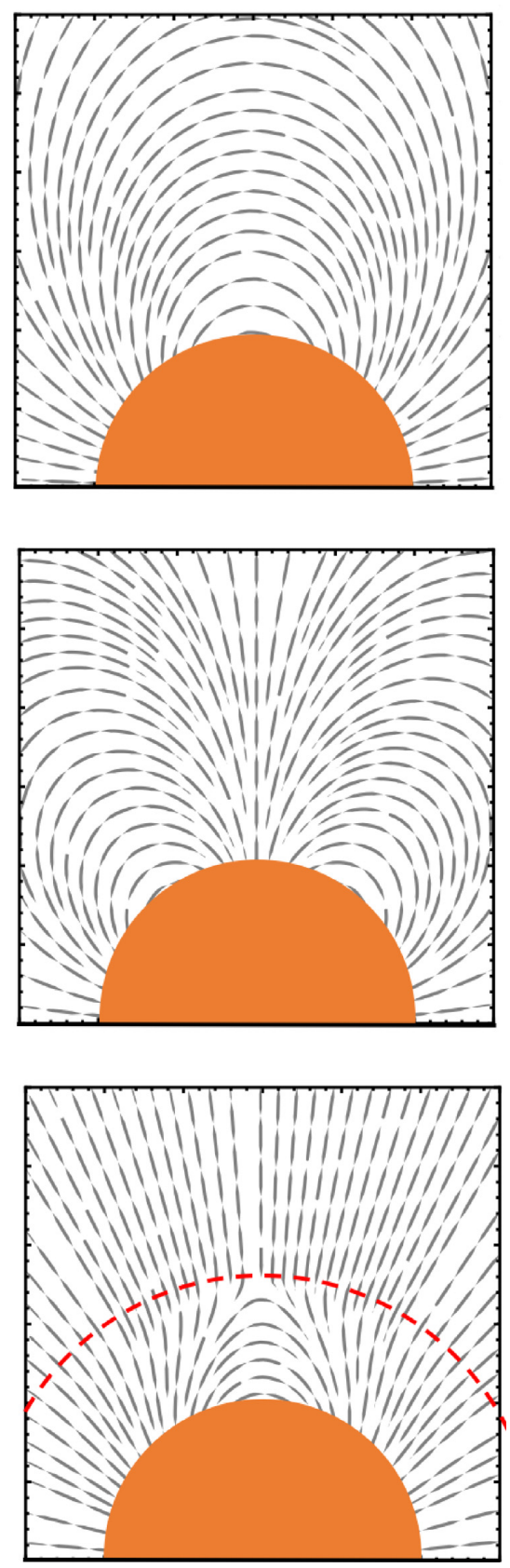

Figure 1. Diagrams of the external magnetic fields used: (top) dipolar, (middle) quadrupolar, and (bottom) dipolar with the addition of a source surface (red dashed line). The stellar surface is shown by the orange circle; this is used throughout the figures in the rest of this paper. The magnetic axis is aligned along the horizontal axis.

we also investigate the impact of allowing the field to be open beyond some radius, known as the 'source surface' $r_{\mathrm{s}}$ (Altschuler $\&$ Newkirk 1969). We focus only on the equatorial plane as shown in Fig. 1 where the field structures are defined by the following equations:

$$
\begin{aligned}
& B_{\mathrm{e}}=B_{0}\left(\left(\frac{2 \cos \phi}{r^{3}}\right) \hat{r}+\left(\frac{\sin \phi}{r^{3}}\right) \hat{\phi}\right), \\
& B_{\mathrm{e}}=B_{0}\left(\left(\frac{3\left(3 \cos (\phi)^{2}-1\right)}{2 r^{4}}\right) \hat{r}+\left(\frac{3 \cos (\phi) \sin (\phi)}{r^{4}}\right) \hat{\phi}\right),
\end{aligned}
$$


and

$B_{\mathrm{e}}=B_{0}\left(\left(\frac{2 \cos \phi}{r^{3}}\right)\left(\frac{r^{3}+2 r_{\mathrm{s}}^{3}}{R_{\star}^{3}+2 r s^{3}}\right) \hat{r}+\left(\frac{\sin \phi}{r^{3}}\right)\left(\frac{-2 r^{3}+2 r_{\mathrm{s}}^{3}}{R_{\star}^{3}+2 r_{\mathrm{s}}^{3}}\right) \hat{\phi}\right)$

for dipolar and quadrupolar fields and a dipolar field that is open beyond $r_{\mathrm{s}}$ (Jardine, Cameron \& Donati 2002a). $B_{0}$ is the maximum magnetic field strength at the stellar surface and $\phi$ represents the angle, measured from the dipole axis $(\phi=0)$, measured in the equatorial plane.

Within this model, we assume that the loops in question are isothermal and 'thin', so that any internal quantities do not vary across the width of the flux tube. In assuming the thin flux tube approximation we also assume that the area of the flux tube is much smaller than the pressure scale height squared, and that the tube does not significantly disturb the background field (Parker 1975; Spruit 1981). They must be in pressure balance with their environment

$B_{\mathrm{i}}^{2}=B_{\mathrm{e}}^{2}+2 \mu\left(p_{\mathrm{e}}-p_{\mathrm{i}}\right)$,

where $\mu$ represents the permeability of free space and $p$ the gas pressure. The subscripts e and i refer to external and internal quantities, respectively. Then, in order to determine the shape of the loops, force balance must be solved along and across the magnetic field lines.

We assume our system to be in hydrostatic equilibrium with no flows, therefore the forces acting on the loop can be modelled by

$\mathbf{0}=-\nabla p+(\mathbf{j} \times \mathbf{B})+\rho \mathbf{g}$,

where $\nabla p$ is the gradient of the gas pressure, $(\mathbf{j} \times \mathbf{B})$ is the Lorentz force, $\rho$ represents the gas density (defined by the equation of state, $p=K_{\mathrm{B}} T \rho / m$, where symbols have the usual meanings and $m$ represents the mean molecular mass) and $\mathbf{g}$ is the gravitational acceleration. Decomposing the Lorentz force into magnetic pressure and tension terms yields

$\mathbf{0}=-\nabla p+(\mathbf{B} \cdot \nabla) \frac{\mathbf{B}}{\mu}-\nabla\left(\frac{B^{2}}{2 \mu}\right)+\rho \mathbf{g}$.

We construct our equations in the co-rotating frame, combining the centrifugal and gravitational forces into an 'effective gravity' term,

$\mathbf{g}_{\mathrm{ef}}=\left(-\frac{G M_{\star}}{r^{2}}+\omega^{2} r\right) \hat{\mathbf{r}}$

which replaces $\mathbf{g}$ in equation (6) and where $\omega$ is the stellar rotation rate. The nature of this equilibrium can be most clearly seen by combining equations (4), (6), and (7) to give

$\mathbf{0}=-\left(\rho_{\mathrm{e}}-\rho_{\mathrm{i}}\right) \mathbf{g}_{\mathrm{ef}}-\nabla\left(\frac{B_{\mathrm{e}}^{2}}{2 \mu}\right)+\left(\mathbf{B}_{\mathrm{i}} \cdot \nabla\right) \frac{\mathbf{B}_{i}}{\mu}$,

where the tension of the loop balances the combined effects of buoyancy and the gradient of the external field. The loop tension is of course determined by its shape. In order to determine this, we decompose equation (6) into components parallel ( $\hat{\mathbf{s}}$ ) and perpendicular ( $\hat{\mathbf{n}})$ to the path of the magnetic field line, we define the unit vectors

$\hat{\mathbf{s}}=\frac{1}{\sqrt{r^{2}+\left(r^{\prime}\right)^{2}}}\left(r^{\prime}, r\right)$

$\hat{\mathbf{n}}=\frac{1}{\sqrt{r^{2}+\left(r^{\prime}\right)^{2}}}\left(-r, r^{\prime}\right)$.

Parallel to the magnetic field line, there is no Lorentz force and equation (6) simplifies to

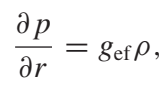

where we have taken the gas pressure ( $p_{0}$ at the stellar surface) to be independent of $\phi$. This defines the gas pressure variation with distance from the surface

$p=p_{0} \exp \left(\frac{m}{K_{\mathrm{B}} T} \int_{R_{\star}}^{r} g_{\mathrm{ef}}(r) \mathrm{d} r\right)$.

The function $H(r)$ can be defined such that

$H(r) \equiv \frac{m}{K_{\mathrm{B}} T_{\mathrm{e}}}\left(-\frac{G M_{\star}}{r}\left(1-\frac{r}{R_{\star}}\right)+\frac{\omega^{2}}{2}\left(r^{2}-R_{\star}^{2}\right)\right)$,

which yields a more compact form of equation (12)

$p_{\mathrm{i}}(r)=p_{0 \mathrm{i}} \exp (H(r))$,

where $p_{0 \mathrm{i}}$ is the base pressure inside the flux tube.

The component of equation (6) perpendicular to the magnetic field is

$0=-\nabla p \cdot \hat{\mathbf{n}}+\left(B^{2} \frac{\partial \hat{\mathbf{s}}}{\partial s}\right) \cdot \hat{\mathbf{n}}+\nabla\left(\frac{B^{2}}{2 \mu}\right) \cdot \hat{\mathbf{n}}+\rho \mathbf{g} \cdot \hat{\mathbf{n}}$,

where

$\frac{\partial}{\partial n}=\hat{\mathbf{n}} \cdot \nabla=\frac{1}{\sqrt{r^{2}+\left(r^{\prime}\right)^{2}}}\left(-r \frac{\partial}{\partial r}+r^{\prime} \frac{\partial}{\partial \phi}\right)$

and

$\frac{\partial \hat{\mathbf{s}}}{\partial s}=(\hat{\mathbf{s}} . \nabla) \hat{\mathbf{s}}=\frac{\left(r r^{\prime \prime}-r^{2}-2\left(r^{\prime}\right)^{2}\right)}{\left.\left(r^{2}+\left(r^{\prime}\right)^{2}\right)\right)^{3 / 2}} \hat{\mathbf{n}}$.

Therefore, the component of the force balance equation perpendicular to the magnetic field line simplifies to

$\frac{2 B_{\mathrm{i}}^{2} r\left(r^{2}+2\left(r^{\prime}\right)^{2}-r r^{\prime \prime}\right)}{\left(r^{2}+\left(r^{\prime}\right)^{2}\right)}=\left(-r^{2} \frac{\partial}{\partial r}+r^{\prime} \frac{\partial}{\partial \phi}\right) B_{\mathrm{i}}^{2}$.

Combining equation (18) with pressure balance as expressed in equation (4), the path of the cooled field line, $r(\phi)$, can be calculated and the loop shape plotted.

\section{LOOP SHAPES}

In exploring the factors that determine the loop shapes we use, as an example, the stellar parameters for a specific star. We choose here to take the parameters of $\mathrm{AB}$ Doradus: $R_{\star}=0.96 \mathrm{R} \odot, M_{\star}=$ $0.86 \mathrm{M}_{\odot}$ and period of $0.514 \mathrm{~d}$ (Innis et al. 1988; Guirado et al. 2010, 2011). We assume the background corona has a temperature of $10^{7} \mathrm{~K}$ (Hussain et al. 2005; Close et al. 2007).

\subsection{Pressure variation with height}

In order to achieve an equilibrium for these loops, two constraints must be satisfied: the internal gas pressure must not become too large relative to its environment and the forces perpendicular to the loop must be in balance. The first constraint is such that in equation (4), $B_{\mathrm{i}}^{2}>0 . B_{\mathrm{e}}^{2}$ will always be positive, however $\left(p_{\mathrm{e}}-p_{\mathrm{i}}\right)$ may be negative if $p_{\mathrm{i}}$ becomes too large. If this difference is small, this need not be a problem for equilibria, so long as $B_{\mathrm{e}}^{2}$ is large enough to balance it. The external field strength decays with height, however, and so beyond a certain value, this will no longer be true and no equilibria may be found. The second constraint corresponds to equation (18), where the magnetic tension (shown on the lefthand side) must be equal to the magnetic pressure gradient (shown on the right-hand side).

Considering the component of force balance along the field line yields equation (14). From this it can be seen how gas pressure 


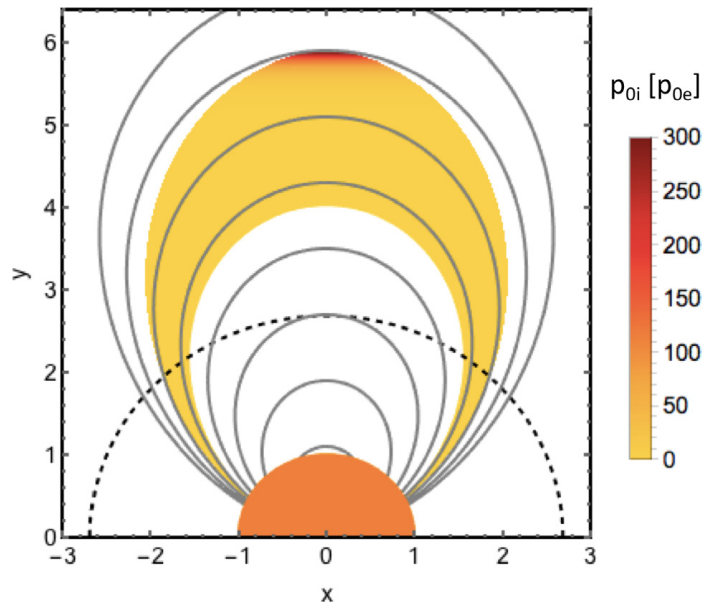

Figure 2. Example of the variation of pressure $\left(p_{\mathrm{i}}\right)$ with height within these cool loops. This is scaled to the external pressure $\left(p_{0 \mathrm{e}}\right)$ at the loop footpoints. This example was calculated with parameters: $T_{\mathrm{e}}=10^{7} \mathrm{~K}, \beta=10^{-7}, p_{0 \mathrm{i}}=$ $2 p_{0 \mathrm{e}}, T_{i}=0.03 \mathrm{~T}_{\mathrm{e}}$. The background field into which this loop would be embedded is shown in grey. The co-rotation radius is shown by the black, dashed line.

varies with height, noting that the sign of $H(r)$ depends on distance from the stellar rotation axis, taking a negative value at low radii and a positive value at high radii. This is dependent on the stellar rotation rate and thus from here the effects of rapid rotation can be seen. Due to the rapid rotation, gas above the co-rotation radius of the star is driven outwards into the tops of loops. Fig. 2 shows an example of the increase in gas pressure with height beyond the co-rotation radius within a magnetic loop. This is similar to the results from Jardine \& Cameron (1991) in a Cartesian geometry, with pressure dropping with height below the co-rotation radius and rising beyond it, as per equation (14).

\subsection{Varying the field topology}

We first choose to vary the field topology, applying both a dipolar and quadrupolar external magnetic field.

An external dipolar field is applied and using equation (18), combined with pressure balance (equation 4 ), solutions can be found for a set of mechanical equilibria. The magnetic field strength is scaled to the external value at the stellar surface. The top panel of Fig. 4 shows the external field in grey on the right-hand side of the figure and some example solutions shown by the blue loops on the left-hand side. The loop shapes deviate from the background field significantly at large heights where the gas pressure dominates over magnetic pressure. The effect of the centrifugal force acting to stretch out the cool, mass-loaded loops can be clearly seen. A 'Height-Width plot' shown in the bottom panel of Fig. 4 shows the family of solutions, with 'height' and 'width' defined in Fig. 3. In this parameter regime, the footpoint separation of the cool loops increases with summit height, following the external field at low radii before deviating from the external field and becoming smaller with increasing loop height as the magnetic field strength drops to zero. It should be noted that 'width' here refers to the angular distance between the loop footpoint and loop summit.

Here we use a very small value of $\beta$ for illustrative effects, to exaggerate the distortion of the loops by the centrifugal effects. When calculating the prominence distributions generated from this model and comparing them to observations, we chose a more real-

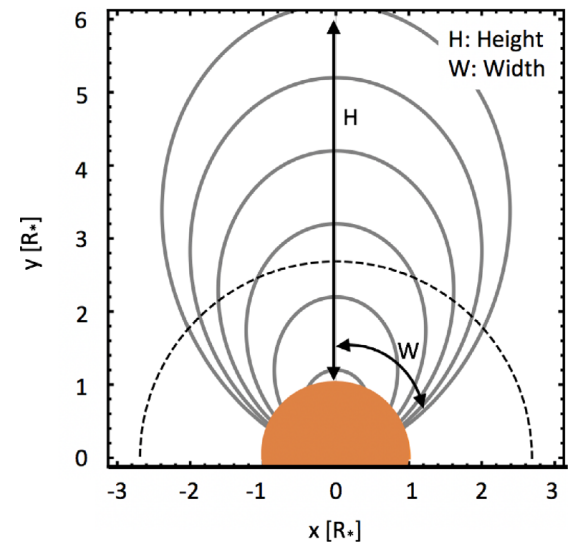

Figure 3. Figure showing the definitions of 'loop height' (or 'distance from the rotation axis') and 'loop width'.

istic value of $\beta$. This value of $\beta$ was viewed as realistic since the average field strength on $\mathrm{AB}$ Dor is observed to be around $10 \mathrm{G}$ and the base pressure can be estimated using $p=\kappa B^{2}=10^{-5.5} B^{2}$ (Jardine et al. 2002b), with $\mathrm{B}$ in Gauss. Thus using $\beta=2 \mu \mathrm{p} / B^{2}$ a value of $10^{-3}$ was seen as reasonable. The effects of varying the base plasma beta value on the maximum loop height was discussed by Jardine \& Cameron (1991), where smaller $\beta$ values were found to allow taller loops. The cooled loops shown in all the examples throughout this paper are only cooled to a tenth of the external field, or $T_{\mathrm{i}}=10^{6} \mathrm{~K}$. This was chosen purely for ease of calculation as dropping the internal temperature can make solutions increasingly difficult to solve for. The effects of varying temperature on these hydrostatic solutions has been discussed previously by Jardine \& van Ballegooijen (2005) and thus was not examined in detail here. Jardine \& van Ballegooijen (2005) considered the effects of decreasing the loop temperature on maximum loop height and found the maximum loop height did not vary significantly between $T_{\mathrm{i}}=$ $10^{6} \mathrm{~K}$ and $T_{\mathrm{i}}=10^{4} \mathrm{~K}$.

An external quadrupolar field is also applied and by the same method, solutions can again be found for a set of mechanical equilibria. An example is shown in Fig. 5 for the same parameters as the above dipolar case. Once again, the cool internal loops are shown in blue on the left-hand side of the top panel and the external field shown on the right-hand side in grey. As with the dipolar field, the family of solutions for the cooled loops follows the external field at low heights, before deviating and becoming taller and thinner with increasing summit height. Naturally, these loops are constrained to a smaller angular extent by the geometry of the system, with the loops now being constrained to $\frac{\pi}{4}$ radians rather than $\frac{\pi}{2}$ radians. This is seen clearly by comparison of the height versus width plots for the two cases.

The maximum attainable loop height can be calculated by considering the point at which the magnetic field of these cool loops $\left(B_{\mathrm{i}}\right)$ vanishes, i.e. where the footpoint separation of the loop is equal to zero. These were found to be $r=5.98 \mathrm{R}_{\star}$ and $r=5.34 \mathrm{R}_{\star}$ for the dipole and quadrupole, respectively. Beyond this height, the tension force cannot increase enough to balance the gradient in magnetic pressure and buoyancy forces, and thus no more hydrostatic solutions can be found.

\subsection{Allowing the field to become open at large heights}

Choosing a background field that has a source surface above the corotation radius, and solving for the shapes of cool loops embedded 

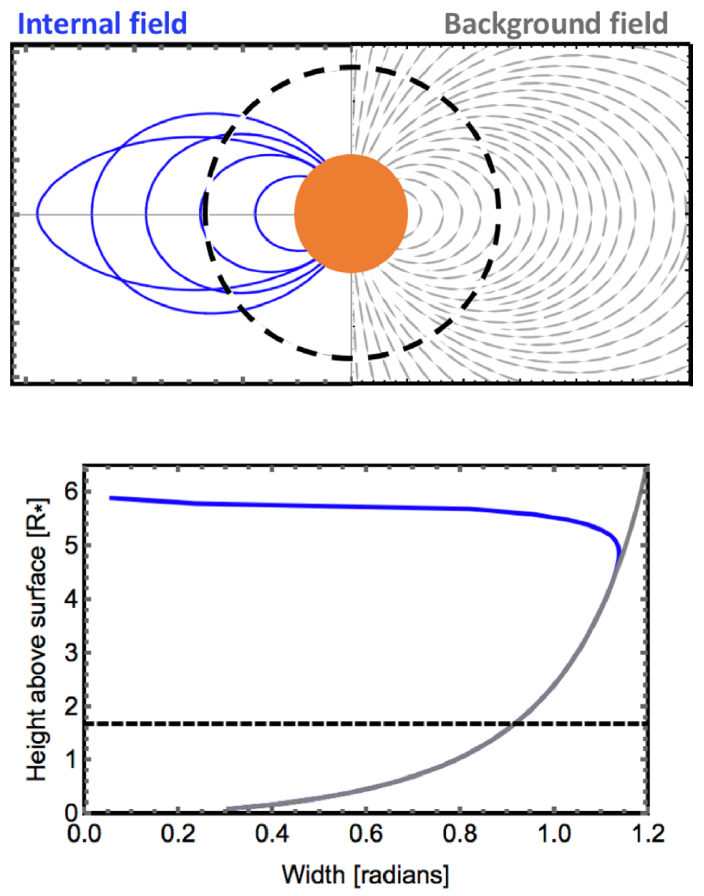

Figure 4. Background (grey) and cooled (blue) loops and the corresponding variation of loop height with footpoint separation (or width) for parameters: $T_{\mathrm{e}}=10^{7} \mathrm{~K}, T_{\mathrm{i}}=T_{\mathrm{e}} / 10, p_{0 \mathrm{i}}=2 p_{0 \mathrm{e}}$ and a plasma $\beta$ at the loop footpoints of $\beta=10^{-7}$. The black dashed line shows the co-rotation radius.
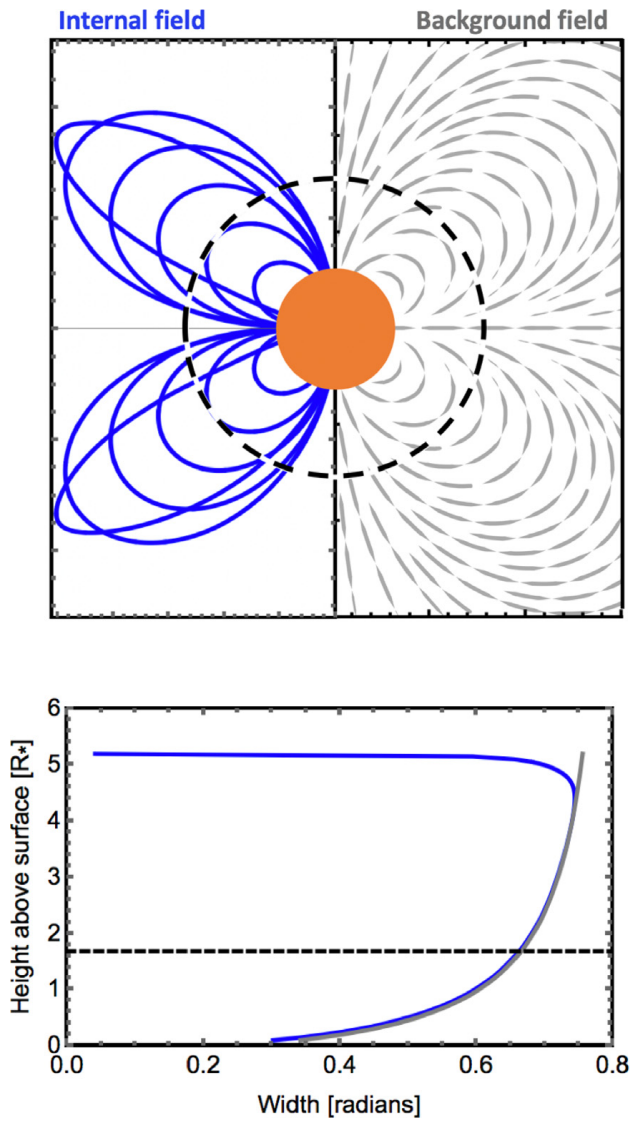

Figure 5. Background (grey) and cooled (blue) loops and corresponding variation of loop height with footpoint separation (or width) for parameters: $T_{\mathrm{e}}=10^{7} \mathrm{~K}, T_{\mathrm{i}}=T_{\mathrm{e}} / 10, p_{0 \mathrm{i}}=2 p_{0 \mathrm{e}}$ and a plasma $\beta$ at the loop footpoints of $\beta=10^{-7}$. The black dashed line shows the co-rotation radius.
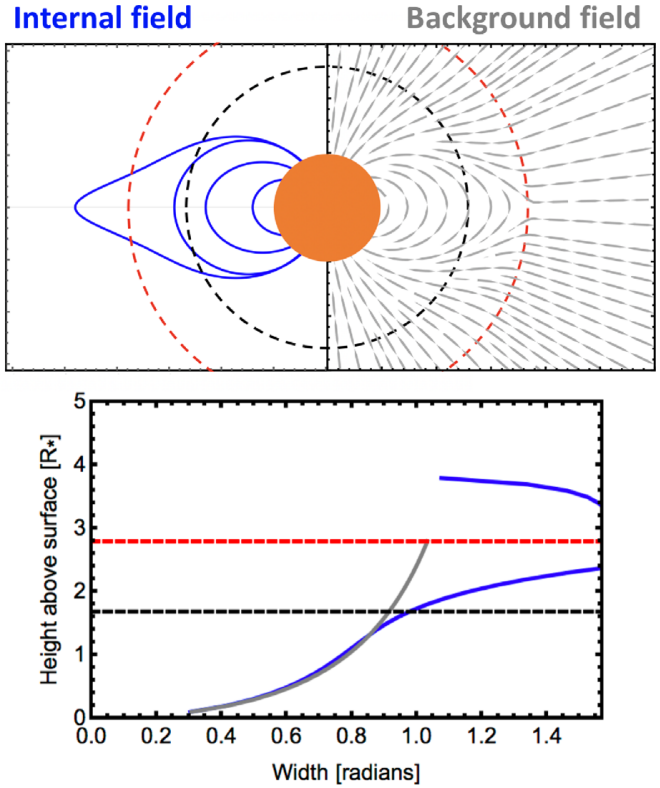

Figure 6. Background (grey) and cooled (blue) loops with inclusion of a source surface (red dashed) and the corresponding variation of loop height with footpoint separation. The co-rotation radius is marked by the black dashed line. The parameters for the cooled loop are $T_{\mathrm{e}}=10^{7} \mathrm{~K}, T_{\mathrm{i}}=T_{\mathrm{e}} / 10$, $p_{0 \mathrm{i}}=11 p_{0 \mathrm{e}}$ and the plasma $\beta$ at the loop base is $\beta=10^{-3}$. The source surface set to $r_{\mathrm{s}}=3.8 \mathrm{R}_{\star}$.

within it, yields Fig. 6. Above the source surface, all external field lines are open, so closed field lines exist in the external field only below this height. As a result, the footpoints of the external field have a maximum separation that depends on the choice of source surface (Jardine et al. 2002a). The tallest closed field line in a dipolar external field connects to the surface at a value of $\phi=\phi_{\max }$ where

$\sin ^{2}\left(\phi_{\max }\right)=\frac{3 r_{\mathrm{s}} R_{\star}}{R_{\star}^{3}+2 r_{\mathrm{s}}^{3}}$.

Hence the maximum distance between footpoints and the summits for the background field is $\left(\pi / 2-\phi_{\max }\right)$. As can be seen in Fig. 6, the curve defining the solutions for the external field terminates at this footpoint separation. For the cooled loops, the solutions for low heights are very similar to those for the external field, but at greater heights their shape differs from that of the external field. Their footpoints can extend beyond the last closed field line in the external region, reaching into the open field region.

Notably, there is a range of heights where no equilibria are available, but a family of equilibria do exist at large heights beyond the source surface, where force balance allows the support of these loops within the stellar wind.

Including a source surface below the co-rotation radius yields Fig. 7. The results for the variation of loop height with footpoint separation are also shown. The loop shapes can be seen to be considerably thinner than the external field at low heights, and once again there is a range of heights around the source surface where no equilibria are available. At greater heights there is a second height range where there are no solutions in this case, and once again there is a family of very tall solutions beyond this.

\subsection{Comparing with observations}

It is clear from the above that there may be several height ranges where no equilibria are available. One is always located around the source surface, and the other at a height where buoyancy and the 
Internal field
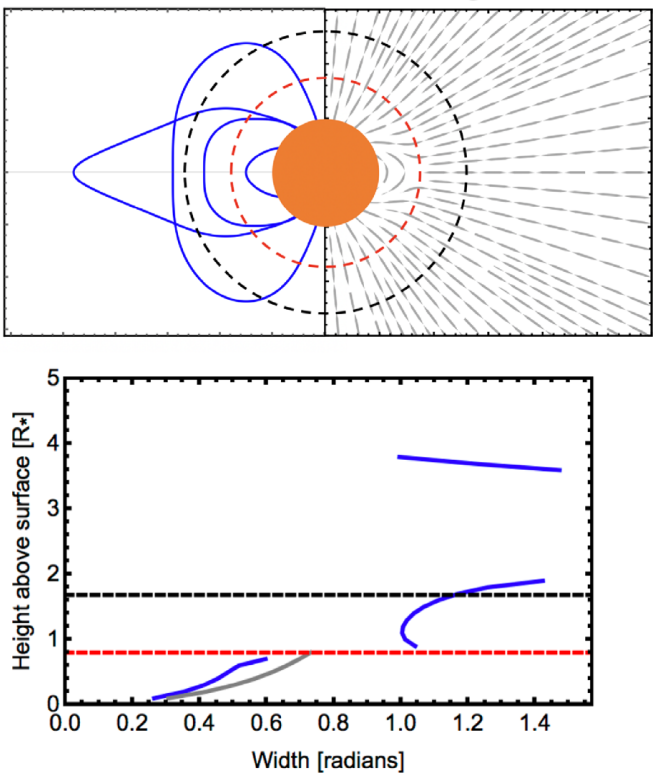

Figure 7. Variation of loop height with footpoint separation for the same parameters as Fig. 6 but now with $r_{\mathrm{s}}=1.8 \mathrm{R}_{\star}$.

upwards pressure gradient of the external field can no longer balance the tension of the cool loop. This latter height range depends on the loop parameters (such as temperature) and the effective surface gravity of the star (which depends on the stellar rotation rate). If a star has loops at a range of temperatures and base pressures, then these 'gaps' in the possible prominence heights may overlap, making it difficult to detect this second gap in the allowed equilibria.

In order to compare these example equilibria with what is observed, we consider two stars for which many prominences have been detected. The two stars selected are AB Dor and BO Mic [also known as Speedy Mic, with spectral type K3V Dunstone et al. (2006a)] and have similar masses but different radii and rotation rates. As a result, their effective gravities and the location of their co-rotation radii are quite different. Comparing these stars therefore allows us to explore the role of the buoyancy force in determining prominence locations. We note that magnetic maps do not exist for Speedy Mic and so we simply assume that the magnetic field is similar to that of $\mathrm{AB}$ Dor. The stellar parameters used for Speedy Mic are $M_{\star}=0.82 \mathrm{M}_{\odot}, R_{\star}=1.06 \mathrm{R}_{\odot}$ and a period of $0.380 \mathrm{~d}$ (Dunstone et al. 2006a).

We have compiled a histogram of the heights of all detected prominences for AB Dor (Cameron \& Robinson 1989; Donati \& Cameron 1997; Cameron et al. 1999; Donati et al. 1999) and Speedy Mic (Dunstone et al. 2006a). These are shown in Fig. 8 for AB Dor in blue and Speedy Mic in green, with their respective co-rotation radii shown by the black dashed lines. Example histograms for predicted prominence heights for typical model parameters are compared to these observations, and shown in grey. These were calculated by sampling the height of loops in increments of $0.2 R_{\star}$ along the Height-Width curves. In Fig. 8, the parameters used are the same as in Fig. 6: $T_{\mathrm{e}}=10^{7} \mathrm{~K}, T_{\mathrm{i}}=T_{\mathrm{e}} / 10, p_{0 \mathrm{i}}=11 p_{0 \mathrm{e}}, \beta=10^{-3}$ and $r_{\mathrm{s}}=$ $3.8 \mathrm{R}_{\star}$. For both stars, our model shows a peak at very low heights, close to the stellar surface, where prominences cannot easily be observed due to their small size, meaning they will not occult a large enough fraction of the star to be easily visible. Their size will be restricted by the magnetic loop constraining them, and thus small loops close to the stellar surface will contain small prominences, which are harder to detect as absorption tracks.

For both stars, a peak is also seen in the model beyond the corotation radius and for $\mathrm{AB}$ Dor a third peak is seen beyond the source surface at around five stellar radii. The gap seen around 3.5 to 4.5 stellar radii from the rotation axis in the histograms for AB Dor corresponds to the gap seen in the Height-Width curves, denoting a transition from magnetically dominated force balance at low heights, to magneto-centrifugal balance at greater heights. These very tall loops can only be supported when the negative buoyancy of the loop is sufficiently large. This can be seen clearly in the plot of the variation of the buoyancy with height (top lefthand panel). In the case of Speedy Mic, in this parameter range, solutions are not found within the stellar wind since the internal magnetic field strength has fallen to zero before the background field has become open.

Considering now the histograms with a lower internal temperature of $T_{\mathrm{i}}=T_{\mathrm{e}} / 15$, the model shows a similar shape of prominence distribution as before but with the peak above co-rotation now shifted to larger heights in both stellar cases. In this parameter range, decreasing the temperature causes the top branch of the Height-Width curve to move outwards from the stellar surface. This can be seen most clearly by considering the force balance shown in equation (8). It is worth noting that despite applying the same magnetic fields to both stars, the loops will experience different buoyancy forces due to the difference in gravitational acceleration on the two stars. This leads to different types of behaviours on the two stars.

While tension always acts inwards and the gradient of the external field acts outwards, the direction of the buoyancy force can change. This term is complicated by the fact that both $\mathbf{g}$ and $\left(\rho_{\mathrm{e}}-\rho_{\mathrm{i}}\right)$ may take positive or negative values. In the case presented here, the Height-Width curve at low heights does not change significantly with a decrease in internal temperature, due to the dominance of the magnetic field. However, the buoyancy force at large heights becomes larger for a decrease in internal temperature, and since for loops of the same width the tension term will be very similar, this requires a smaller gradient of external field term in order to still reach an equilibrium. This results in the top branch of the curve moving outwards, and thus the final peak in the distribution also moving to larger heights. It should be noted that this behaviour will not always be seen, as it depends on the parameter regime in which we find ourselves, due to the complexity of the buoyancy term.

Speedy Mic. still cannot support prominences within the open field with these parameters, though again the peak of the distribution has shifted outwards. The buoyancy term here is more straightforward since for these parameters the term only changes sign at the co-rotation radius i.e. when the effective gravity changes sign. However, we find ourselves in a similar situation as the buoyancy term acts outwards and becomes larger with decreasing temperature, thus once again the peak of the distribution moves outwards.

Plotting prominence distributions for a source surface below corotation, with parameters as Fig. 7, results in rather different behaviour, as shown in Fig. 9. For AB Dor the gap in the Height-Width curve shown in Fig. 7 can be seen clearly, in addition to another gap around the source surface. For Speedy Mic however, the rapid rise in the magnitude of the buoyancy above the co-rotation radius cannot be balanced by the loop tension without the added effect of the upwards pressure gradient of the external field. In this case, 


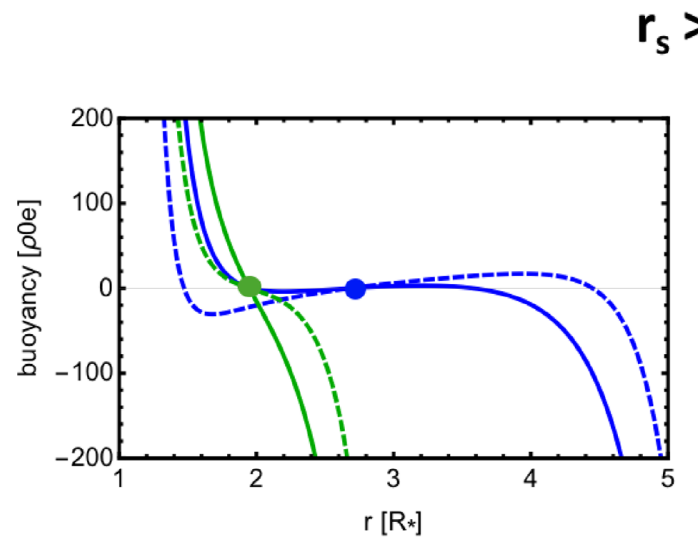

$r_{s}>r_{k}$
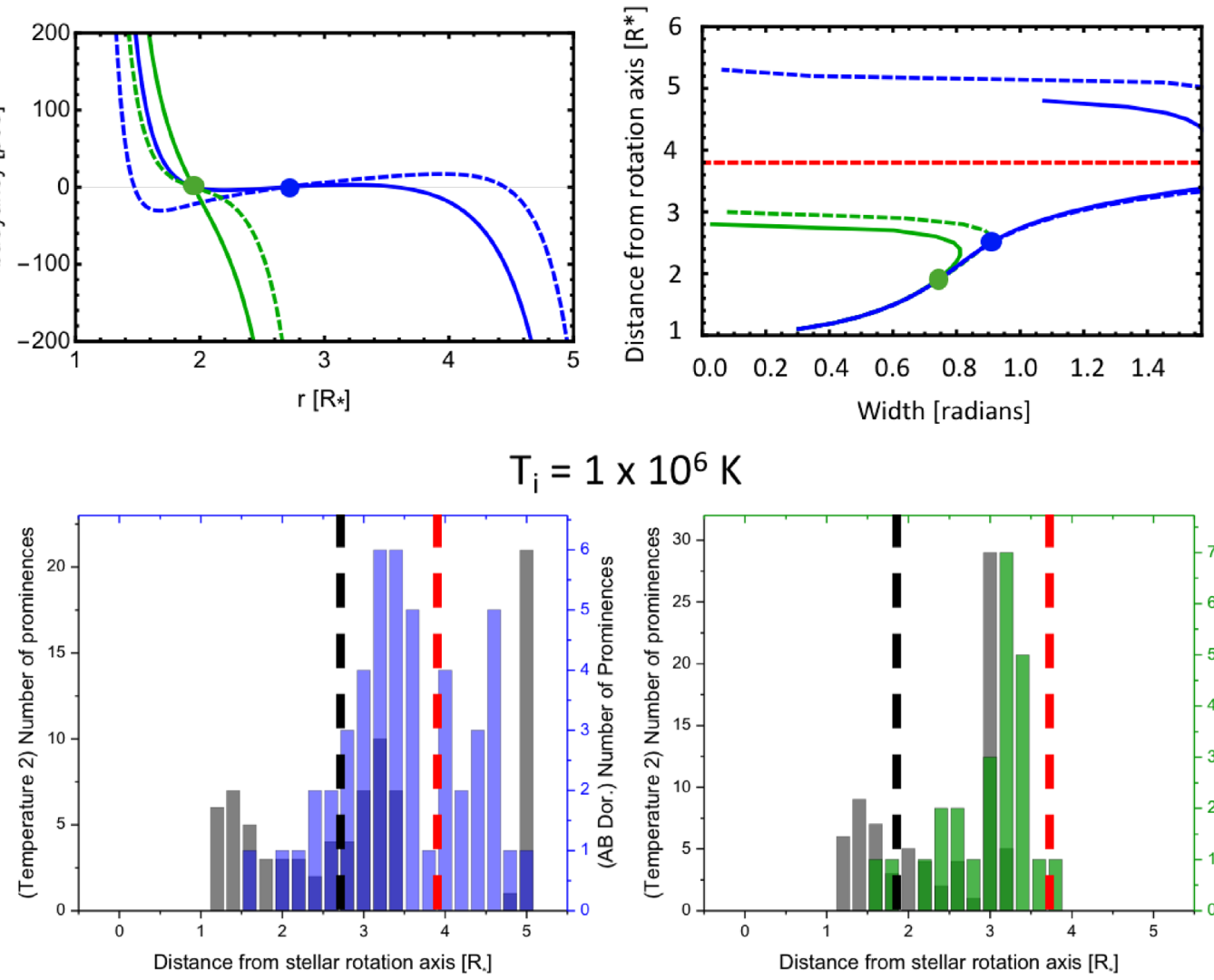

$\times 10^{6} \mathrm{~K}$



Distance from stellar rotation axis $[R$.]

AB Dor observations

model

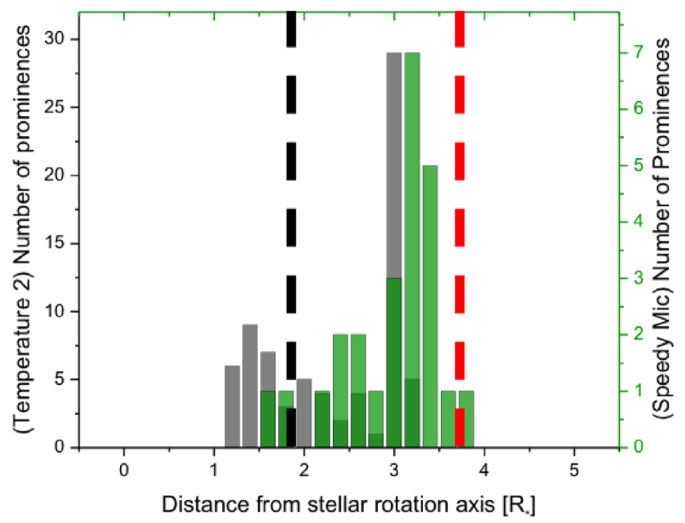

Figure 8. Top Panel: Buoyancy $\left(\rho_{\mathrm{e}}-\rho_{\mathrm{i}}\right) / g_{\mathrm{ef}}$ (left) and Height-Width plots (right) for AB Doradus (blue) and Speedy Mic (green). The co-rotation radii for the stars are shown by dots. Middle and Bottom Panels: Histograms of prominence observations for the two stars and example distributions in grey for an internal temperature of $T_{\mathrm{i}}=T_{\mathrm{e}} / 10$ and $T_{\mathrm{i}}=T_{\mathrm{e}} / 15$. The co-rotation radius and source surface $\left(r_{\mathrm{s}}=3.8 \mathrm{R}_{\star}\right)$ are shown by the black and red dashed lines, respectively. Parameters here are as Fig. 6.

because the field is open above the co-rotation radius, it is impossible to achieve force balance for heights significantly above co-rotation and so the distribution of model equilibrium heights is extremely compact.

\section{DISCUSSION}

The effective gravity of a star, and in particular its rotation rate, clearly has a strong influence on the equilibrium summit heights of loops that have cooled. The geometry of the background field may 


\section{$r_{s}<r_{k}$}
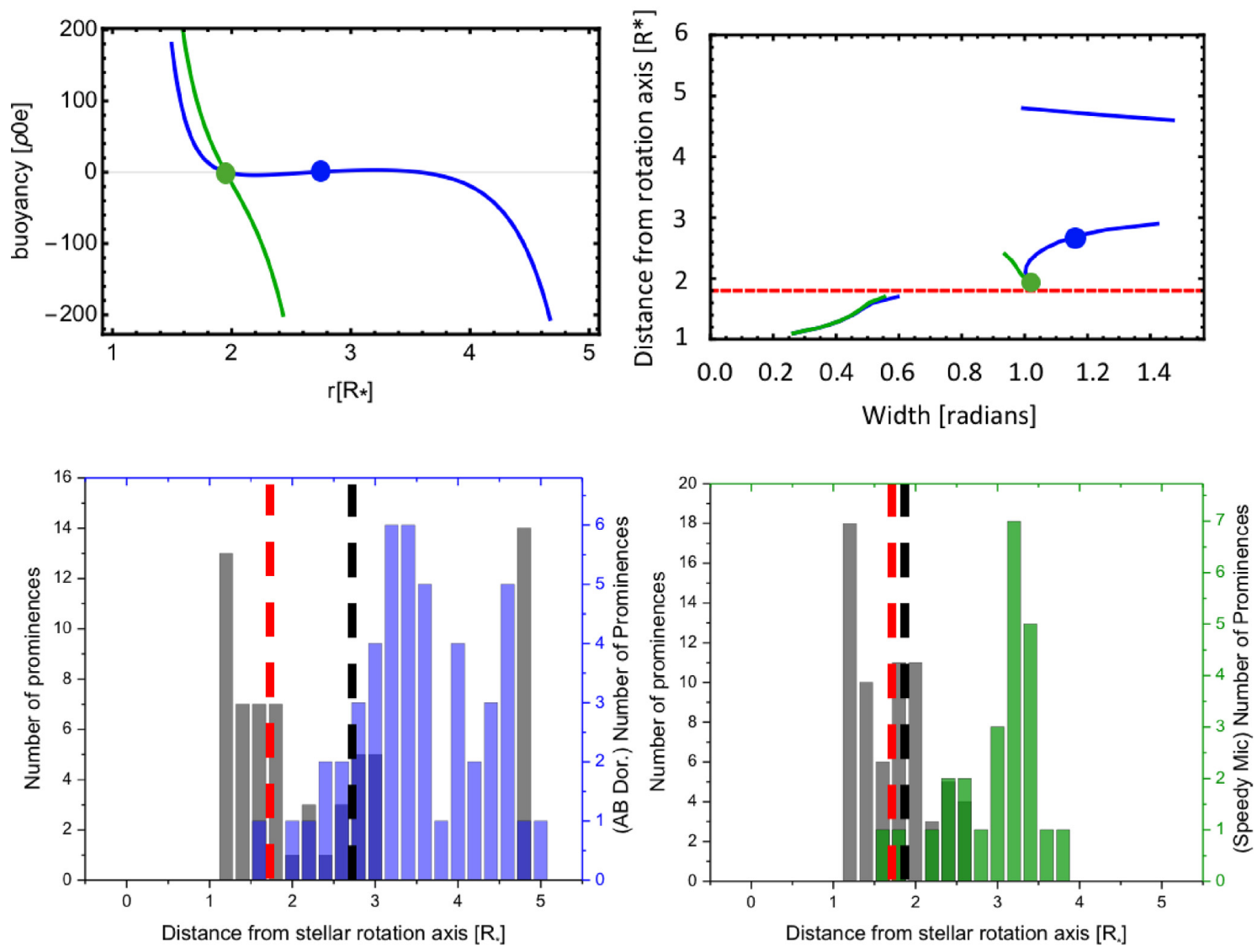

AB Dor observations

Speedy Mic observations

model

model

Figure 9. Top Panel: Buoyancy (left) and Height-Width plots (right) for AB Doradus (blue) and Speedy Mic (green). The co-rotation radii for the stars are shown by dots. Bottom Panel: Histograms of prominence observations for the two stars and example distributions in grey for an internal temperature of $T_{\mathrm{i}}=$ $T_{\mathrm{e}} / 10$. The co-rotation radius and source surface $\left(r_{\mathrm{s}}=1.8 \mathrm{R}_{\star}\right)$ are shown by the black and red dashed lines, respectively. Parameters here are as Fig. 6.

also play a role, however, of particular important is the radius at which this background field becomes open.

\subsection{Changing the field topology}

With this model we have prescribed both dipolar and quadrupolar stellar fields. These are the two lowest order terms in the multipolar expansion for the overall structure of the stellar magnetic field and are the ones most likely to dominate at the large heights at which prominences are observed. The quadrupolar field would naturally result in prominences of smaller angular extent. This topology would also be able to explain the presence of four prominences in each hemisphere at one time, as opposed to the two prominences from the dipolar field.

The two multipoles examined here also show slight variation in the maximum attainable height of these loops, with $r=5.98 \mathrm{R}_{\star}$ and $r=5.34 \mathrm{R}_{\star}$ for the dipole and quadrupole, respectively. This is explained by the fact that the prescribed quadrupolar magnetic field falls off more rapidly with height than the dipolar field. The difference in maximum height is small, and it is unlikely that from observed prominence heights alone one would be able to predict the loop topology that best describes the prominence hosting loop. This maximum height is also dependent on the loop parameters, which cannot be obtained directly from observational data.

Although results have only been shown for a dipole and quadrupole external field, in principle any external magnetic field may be prescribed here. This could include higher order multipole expansions, a combination of the multipole expansions, or an entirely different external field.

\subsection{Inclusion of a source surface}

Results here replicate the essential conclusion of Jardine \& van Ballegooijen (2005) that prominences may be supported within the open field region, i.e. within the stellar wind. We find equilibria for source surfaces both above and below the co-rotation radius, and in both cases 'helmet streamer' loop shapes can be found. Solutions around the source surface are, however, difficult to find.

In the case of $r_{\mathrm{s}}>r_{\mathrm{k}}$, the Height-Width curve generated is similar in form to that of the pure dipole case with an upper and lower 
branch, separated by a gap around the location of the source surface. Once the source surface is brought inside of co-rotation, however, (Fig. 7) the family of solutions now shows a middle branch, with gaps above and below it, although it still retains the same overall shape. Loops in this case have considerably flatter loop summits, in order to balance the forces acting on them, than is seen in the other cases presented here.

The location of the gap in equilibria due to the growth in the buoyancy force above co-rotation depends on the loop parameters (such as the temperature and field strength) and the stellar parameters (principally the effective gravity). Its presence could therefore be difficult to detect by compiling a histogram of all observed prominences, since they may have a variety of parameters. The gap due to the location of the source surface is at the same height for all loops however.

\subsection{Development from previous models}

One of the main limitations of Jardine \& van Ballegooijen (2005) was that it was restricted to a Cartesian geometry. It is therefore most suitable for small scales where the star is locally flat. By extending this to a spherical geometry, we have been able to allow for the global field structure of star. In addition, we have removed the infinite horizontal domain implicit in the earlier models. In the present model, loop footpoints may only extend to $\pi$ radians in the dipolar case and $\pi / 2$ radians in the quadrupolar case.

As a result of the Cartesian geometry, the solutions of Jardine \& van Ballegooijen (2005) did not allow equilibria at the co-rotation radius, if this was above the source surface (i.e. in the open field region). The reason is that at the co-rotation radius, the effective gravity, and hence the buoyancy force, is zero. In a Cartesian geometry, however, the field lines in the wind region are straight and purely vertical and so there is no gradient in the magnetic pressure to balance the loop tension. As a result, no equilibria exist. In a spherical geometry, the magnetic pressure gradient does not vanish in the radial field of the wind and so equilibria may still exist at the co-rotation radius.

In principle, the source surface model here does not require a specific prescribed external field, as is the case in the Cartesian model, and various multipoles could be prescribed in the place of the dipole. This model is still restricted to the equatorial plane, through our definition of the effective gravity term, and development of this into a fully three-dimensional model would be interesting but complex.

\subsection{Interpreting the observed distributions of prominence heights}

The aim of this work is not to attempt to reproduce the observed distributions of prominence heights in detail, but to shed light on their overall structure. The observations reveal the location of large prominences that form at heights of several stellar radii. Smaller, more low-lying prominences (such as those predicted by our models) may be present, but they would not produce sufficiently large absorption signatures to be detected. We therefore focus on the height distributions of the larger prominences that are supported at greater heights.

Our analysis reveals that there may be two gaps in the prominence distribution, caused by two different physical effects. The first is the location of the source surface, where the field transitions from closed to open. This is at a fixed location in our model. The second gap is due to the behaviour of the buoyancy, which for the tallest equilibria is the dominant force opposing the tension of the cool loop. As can be seen from Fig. 8, this force behaves quite differently in our two example stars. For Speedy Mic the co-rotation radius is much closer to the stellar surface, with the result that centrifugal effects dominate at lower heights. This leads to a more compact distribution of equilibria, regardless of the location of the source surface. The buoyancy force depends not only on the effective gravity, which is a global stellar parameter, but also on the local parameters (such as the temperature) for each cool loop. We show in Fig. 8 the effect on this term of a change in the temperature. Although this is less significant than the difference between the two stars, it can still change the location and width of the gap in loop equilibria. Consequently, if a star has cool loops at a range of temperatures, any gaps due to buoyancy effects may be smeared out in the distribution of observed prominence heights.

It should also be noted that the histogram for the observed data for Speedy Mic came from one observing run, with only a few data points, while the data used for $\mathrm{AB}$ Dor came from a $10 \mathrm{yr}$ period. The magnetic field structure for AB Dor had changed over this time period (varying by an order of magnitude over $10 \mathrm{yr}$, corresponding to a change in $\beta$ of 0.01 ) and thus the histogram plotted may in fact be a combination of multiple histograms. We do not have X-ray data over this extended period and so we do not know if the coronal temperature varied. Extended observations of a star, long enough to include a meaningful number of data points and catch prominences at all heights, taken over a shorter time period than the data used here for $\mathrm{AB}$ Dor would be needed in order to better compare the model to data.

\section{SUMMARY AND CONCLUSIONS}

We present a model for calculating mechanical equilibrium of magnetic loops for rapidly rotating stars in a spherical geometry. We model families of solutions for loops embedded in a background field that is either a dipolar or quadrupolar geometry, showing that the model is able to adapt to different stellar magnetic field topologies. We have included the effect of the magnetic field becoming open beyond some radius (the source surface), both within and outside of co-rotation, and solutions have been found in the open field region as in Jardine \& van Ballegooijen (2005). Using these solutions, we show histograms of prominence heights for various prominence temperatures and compare to data from AB Dor and Speedy Mic. The model is able to predict the peak of prominences above the co-rotation radius, but also predicts many prominences at low heights, where they cannot be detected by current methods.

Unlike the Cartesian model previously presented by Jardine \& van Ballegooijen (2005), our spherical geometry allows for equilibria that pass smoothly through the co-rotation radius. This matches the observational data from $\mathrm{AB}$ Dor. Looking at the Height-Width curves, we notice that lower solutions are almost force free, following the external field very closely, before the buoyancy term becomes important and can no longer be ignored, ultimately determining the maximum height once the force has become too large to be balanced. We find that the exact balancing of these forces is complex, based on the buoyancy force which may act outwards or inwards depending on both the direction of the effective gravity and the sign of the density difference. By comparison of the observed prominence distributions, we expect Speedy Mic to have a more compact corona than $\mathrm{AB}$ Dor, since a third peak of prominences is not seen here, which is consistent with the stronger effective gravity force of this star due to its larger radius and shorter rotation period. We note that for both stars the overall shape of the height distribu- 
tions is closer to that observed when the opening radius is beyond the co-rotation radius.

We have identified two physical processes that can produce gaps in the height distribution of prominences. The first is the opening up of field lines at the source surface and the second is the magnitude and direction in which the buoyancy force acts. This second process will vary from loop to loop if individual loops are at different temperatures and this may act to disguise the presence of this gap in distributions of prominences for an entire star. The location of the source surface may be more robust to variations from loop to loop and so this may be detectable in distributions of observed prominence heights if these are acquired over a short enough time period that the background stellar field does not evolve.

\section{ACKNOWLEDGEMENTS}

The authors would like to thank the referee for helpful comments that improved the paper and acknowledge support from STFC.

\section{REFERENCES}

Aarnio A. N., Matt S. P., Strassun K. G., 2012, ApJ, 760, 1

Altschuler M. D., Newkirk G., 1969, Sol. Phys., 9, 131

Barnes J. R., Cameron A. C., Unruh Y. C., Donati J.-F., Hussain G. A. J., 1998, MNRAS, 299, 904

Barnes J. R., Cameron A. C., James D. J., Donati J.-F., 2000, MNRAS, 314, 162

Byrne P. B., Eibe M. T., Rolleston W. R. J., 1996, A\&A, 311, 651

Cameron A. C. et al., 1999, MNRAS, 308, 493

Cameron A. C., 1996, International Astronomical Union. Kluwer Academic Publishers, Dordrecht, p. 449

Cameron A. C., 1999, ASP Conf. Ser., 158, 146

Cameron A. C., Robinson R., 1989, MNRAS, 236, 57

Cameron A. C., Woods J. A., 1992, MNRAS, 258, 360

Cassinelli J. P., Brown J. C., Maheswaran M., Miller N. A., Telfer D. C., 2002, ApJ, 578, 951

Close L. M., Thatte N., Nielsen E. L., Abuter R., Clarke F., Tecza M., 2007, ApJ, 665, 736

Cranmer S. R., 2017, ApJ, 840, 1

Donati J.-F., Cameron A. C., 1997, MNRAS, 291, 1

Donati J.-F., Landstreet J., 2009, ARAA, 47, 333

Donati J.-F., Cameron A. C., Hussain G. A. J., Semel M., 1999, MNRAS, 302,437

Dunstone N. J., Barnes J. R., Cameron A. C., Jardine M., 2006a, MNRAS, 365,530

Dunstone N. J., Cameron A. C., Barnes J. R., Jardine M., 2006b, MNRAS, 373,1308

Eibe M., 1998, A\&A, 337, 757

Ferreira J. M., 2000, MNRAS, 316, 647
Gonzalez W. D., Joselyn J. A., Kamide Y., H. W. Kroehl G. R., Tsuruani B. T., Vasyliunas V. M., 1994, J. Geophys. Res., 99, 5771

Guirado J. C. et al., 2010, ASSSP, Highlights of Spanish Astrophysics V. Springer-Verlag, Berlin Heidelberg,p. 139

Guirado J. C., Marcaide J. M., Marti-Vidal I., Le Bouquin J. B., Close L. M., Cotton W. D., Montalban J., 2011, A\&A, 533, A106

Hall J. C., Ramsey L. W., 1992, AJ, 104, 1942

Hall J. C., Huenemoerder D. P., Ramsey L. W., Buzasi D. L., 1990, A\&A, 358,610

Hussain G. A. J. et al., 2005, ApJ, 621, 999

, Hussain G. A. J., 2013, in Brigitte S., Jean-Marie M., S.T Wu, eds, Proc. IAU Symp. 300, Observations of stellar coronae and prominences. Kluwer, Dordrecht, p. 309

Innis J. L., Thompson K., Coates D. W., Lloyd Evans T., 1988, MNRAS, 235,1411

Jardine M., Cameron A. C., 1991, Sol. Phys., 131, 269

Jardine M., van Ballegooijen A., 2005, MNRAS, 361, 1173

Jardine M., Cameron A. C., Donati J.-F., 2002a, MNRAS, 333, 339

Jardine M., Wood K., Cameron A. C., Donati J.-F., Mackay D. H., 2002b, MNRAS, 336, 1364

Jeffries R. D., 1993, MNRAS, 262, 369

Khodachenko M. L. et al., 2007, Astrobiol., 7, 167

Leitzinger M., Odert P., Zaqarashvili T. V., Hanslmeier R. G. A., Lammer H., 2016, MNRAS, 463, 965

Low B., 1994, Phys. of Plas., 1, 1684

Low B. C., 2006, ApJ, 646, 1288

Odert P., Leitzinger M., Hanslmeier A., Lammer H., 2017, MNRAS, 472, 876

Owocki S. P., ud-Doula A., Townsend R. H. D., Petit V., Sundqvist J. O., Cohen D. H., 2016, MNRAS, 462, 3830

Parker E. N., 1975, ApJ, 201, 494

Petit V. et al., 2013, MNRAS, 429, 398

Schroder K.-P., 1983, A\&A, 124, 16

Skelly M., Unruh Y., Cameron A. C., Barnes J., Donati J.-F., Lawson W. Carter B., 2008, MNRAS, 385, 708

Skelly M. B., Unruh Y. C., Barnes J. R., Lawson W. A., Donati J.-F., Cameron A. C., 2009, MNRAS, 399, 1829

Skelly M. B., Donati J. F., Bouvier J., Grankin K. N., Unruh Y. C., Artemenko S. A., Petrov P., 2010, MNRAS, 403, 159

Spruit H. C., 1981, A\&A, 98, 155

Stauffer J. et al., 2017, AJ, 153, 152

Steeghs D., Horne K., Marsh T. R., Donati J. F., 1996, MNRAS, 281, 626

Townsend R. H. D., Owocki S. P., 2005, MNRAS, 357, 251

Ud-Doula A., Owocki S. P., Townsend R. H. D., 2008, MNRAS, 385, 97

Vida K. et al., 2016, A\&A, 590, A11

Villarreal D'Angelo C., Jardine M., See V., 2018, MNRAS, 475, L25

Wang J., Zhou G., Zhang J., 2004, ApJ, 615, 1021

This paper has been typeset from a $\mathrm{T}_{\mathrm{E}} \mathrm{X} / \mathrm{LT} \mathrm{T} \mathrm{X}$ file prepared by the author. 